\title{
Game Theoretical Aspects of the Minds of Gods
}

\author{
Aaron D. Lightner*a,b and Benjamin Grant Purzycki ${ }^{\mathrm{a}}$ \\ ${ }^{a}$ Department of the Study of Religion, Aarhus University, DK \\ ${ }^{\mathrm{b}}$ Department of Anthropology, Washington State University, USA
}

\begin{abstract}
From Pascal's Wager and Guthrie's theory of religion as anthropomorphism to supernatural punishment and the distribution of ritual centers, elements of game theory have been useful for framing various dilemmas that people face between each other and their deities. This chapter first provides a brief introduction to game theory and discusses how it has been used to address various questions in the context of religion and cooperation. It then concludes with a brief discussion of how future research might use it to investigate particular religious traditions.
\end{abstract}

\section{Elements of game theory}

Game theory is about understanding strategic interactions (Maynard Smith, 1982; Von Neumann and Morgenstern, 1953). Models in game theory are abstract and powerful tools that we can use to clarify a wide range of questions in the social and biological sciences, such as why male deer walk parallel to each other before a fight or why oak trees in the same forest grow to about the same height (Kokko, 2007; McNamara and Leimar, 2020). As we will discuss in this chapter, game theory is quite useful for understanding a wide range of dilemmas that are central to religious beliefs and practices (Bulbulia, 2012; Purzycki and Sosis, 2022).

To model an interaction as a game is to represent and analyze a structured scenario among multiple actors, whose actions affect the outcomes of the others. The outcome of a game is a set of payoffs to each actor, and these depend on the actors' chosen strategies. Game theoretical models can help us make predictions in otherwise unpredictable scenarios, but this requires some starting assumptions about how each actor will select a strategy. A common assumption, for example, is that each actor is self-interested and rational, and will therefore select their strategies based on expected utility (a general-purpose term of payoffs).

To see this, consider a game with two actors - let's call them Alice and Bob - who we assume will each choose the strategy that maximizes his or her expected payoff. This assumption not only helps us predict how they will choose their strategies, but how they will each respond to available information about how the other might behave. If Alice knows the strategies and payoffs available to Bob, then based on this knowledge she can choose the strategy that will produce her most favorable possible outcome. In this scenario, we can say that Alice has chosen a best response.

Alice might not always have complete knowledge about Bob's strategies and payoffs, however, meaning she is acting on incomplete information. If so, and if the game is repeated, then Alice and Bob might each learn from previous interactions to converge onto their own best responses (Binmore, 2011). When Alice and Bob have each selected a best response, and neither can improve

\footnotetext{
*Corresponding author: adlightner@cas.au.dk
} 
their payoffs by unilaterally switching strategies, then the outcome is called a Nash equilibrium (Nash, 1950).

In a game, strategies and their associated payoffs might not only reflect people's preferences, but they can also represent organizational policies, social norms, or even genetically evolved traits. The latter applies to evolutionary game theory, which, as we discuss below, operates under a slightly different set of assumptions and concepts while bearing key similarities to standard game theory.

Game theoretic interactions are often characterized as competitive, but as we discuss next, most realistic game scenarios have the potential for both conflict and coordination, regardless of how we conceptualize strategies and utility.

\subsection{Equilibria in games of conflict and coordination}

In Conan Doyle's "The Final Problem," (Doyle, 2003, pp. 736-755) a train scene occurs in which Professor Moriarty wishes to murder Sherlock Holmes while pursuing him from London to Dover. Holmes knows that Moriarty knows his destination, and vice versa, meaning that if Holmes departs at Canterbury, the intermediate stop, then Moriarty can anticipate this plan and also depart at Canterbury to accost Holmes. This might imply that Holmes should go to Dover as planned. And yet, Moriarty can also anticipate this alternative to his departure at Canterbury, implying that Holmes should, in fact, go to Canterbury (and so on, ad infinitum).

This simple scenario is an example of a game of pure conflict, because the strategies (depart at Canterbury or depart at Dover) available to each actor (Holmes and Moriarty) entail strictly opposing interests: Moriarty wishes to depart at the same stop as Holmes, and Holmes wishes that he and Moriarty will depart at different stops. Table 1 illustrates a payoff matrix of this dilemma, where each cell includes Holmes' and Moriarty's payoffs.

Table 1: Payoffs in a game of pure conflict

\begin{tabular}{ccc}
\hline \hline & \multicolumn{2}{c}{ Moriarty } \\
Holmes & Canterbury & Dover \\
\hline Canterbury & 0,1 & 1,0 \\
Dover & 1,0 & 0,1 \\
\hline \hline
\end{tabular}

Now consider a different scenario: Alice and Bob are each driving on the same path toward each other, with a complete absence of traffic laws. If we are assuming that Alice and Bob are each rational, self-interested utility maximizers, then we might assume that each prefers to avoid a collision. Hence, this Driving Game has at least two equilibria: Alice and Bob each choose to drive on their left, or they each choose to drive on their right (Binmore, 1994). This simple scenario is an example of pure coordination, because the strategies (drive to the left or drive to the right) available to Alice and Bob must match for each actor to maximize his and her expected payoffs (table 2).

Table 2: Payoffs in a game of pure coordination

\begin{tabular}{ccc}
\hline \hline \multirow{2}{*}{ Alice } & \multicolumn{2}{c}{ Bob } \\
\hline Drive on the left & 1,1 & 0,0 \\
Drive on the right & 0,0 & 1,1 \\
\hline \hline
\end{tabular}

Coordination solutions to repeated games, such as the equilibria in the Driving Game, can lead to social conventions (Lewis, 1969). If Alice encounters Bob in a future driving scenario, and her past encounter leads her to believe that Bob will drive to the left, then her best response is to also drive to the left. If this type of encounter is repeated further, then over time both players can converge onto a default rule, or convention, such as "always drive on the left." Whenever Alice 
and Bob recognize this coordination problem in the future, they can both easily remember their convention for avoiding collisions, thereby solving their recurring coordination problem.

In a society, conventions can be codified into laws, norms, or ritual practices. They are stabilized by incentives, beliefs about other people's incentives, and a continuing preference for the coordination solution, such as avoiding collisions (Yamagishi and Suzuki, 2009). No particular outcome is needed, so long as the convention is an equilibrium that continues delivering on everyone's preferences (Binmore, 2011).

Game theory provides us with a powerful formal framework for representing a variety of dilemmas, where payoffs and actions matter to the individuals involved. The games we will discuss not only capture varying types of conflict and coordination as we described here, but they can also be useful for modeling dilemmas ranging from whether or not one would believe in god to how and why people are motivated to participate in religious rituals.

\section{Minds of gods and games against nature}

In his posthumous collection of fragments (Pensées), mathematician and theologian Blaise Pascal argued that belief in God is a rational and pragmatic decision, given the expected payoffs for believing versus disbelieving (§233). Conceptualizing belief in God as a decision under risk, Pascal noted that the possible errors have asymmetric payoffs: a true positive is infinitely beneficial (i.e., heaven), but a false positive is a trivial error. Not believing when there is a god, however, entails eternal damnation. Table 3 spells out a candidate believer's possible payoffs to Pascal's Wager. The strategy individuals must choose is to believe or not believe, whereas the "strategy" imposed by nature is a hidden property about the universe: God either exists or does not exist (for a more extensive game theoretical analysis of this and other theological dilemmas, see Brams, 1983, 2018).

\begin{tabular}{ccc} 
Table 3: Pascal's Wager Payoff Matrix \\
\hline \hline \multicolumn{3}{c}{ God Exist? } \\
Believe? & Yes & No \\
\hline Yes & Eternal Bliss & Oops \\
No & Eternal Damnation & Bragging Rights \\
\hline \hline
\end{tabular}

Pascal's wager may be a problematic argument for a variety of philosophical and psychological reasons (e.g., can we safely assume that we choose our beliefs, or that there are virtually no costs to believing? See Brams, 1982; Hacking, 1972, for further discussion), but its underlying logic foreshadows a recurring theme among so-called evolutionary games against nature. That is, organisms in the natural world routinely must deal with fitness-relevant tasks that carry highly asymmetric costs and benefits among their possible errors (Haselton et al., 2009). Correct inferences have their benefits, but a failure to detect a hidden fact about the world can be devastating.

\subsection{Superstitions and faces in the clouds}

Genetically evolved traits are a bit like hypotheses about the environment. Under high levels of uncertainty, the success of a trait depends on it making a fitness-relevant bet about the environment's structure, and ultimately, being less wrong than other possibilities. We can therefore combine game theory with biological principles to usefully model the evolutionary process as different species' traits playing against the environment in different games against nature.

In evolutionary game theory's earlier days, theorists were interested in how evolved strategies (traits) were selected, where the relevant utility was not a preference among decision-makers, but the probability of a strategy's continued survival. Hence, a higher utility would reflect a heritable strategy that properly hedges its bets against nature's "strategies," or the uncertain properties of 
the environment that might, with some probability, lead to a species' extinction (Lewontin, 1961). This is a key difference in assumptions between evolutionary game theory vs. standard game theory: strategies are not selected by a principle of rational choice, but as a consequence of natural selection (Maynard Smith, 1982).

This is especially important when we consider how individuals' preferences can diverge from their fitness-relevant interests. We might strongly prefer true inferences over false ones, for example, but our evolved intuitions are ultimately shaped by how they affect fitness, and not by how true they are (Prakash et al., 2021). True inferences are, of course, frequently useful for improving fitness, but only insofar as they serve our ability to make useful predictions that are both important to survival and able to handle some degree of error. Always making perfectly accurate decisions might intuitively seem like the optimal policy, but doing so is impractical, inefficient, and computationally intractable under even marginally complex and dynamic conditions (Bossaerts and Murawski, 2017). It is often the case that practical and ecologically rational decision heuristics outperform more sophisticated decision strategies in highly uncertain but structured environments (Gigerenzer et al., 2011).

Consider the prevalence of superstitions, or mistaken inferences about unknown cause-effect relationships. Superstitious beliefs and behaviors play a central role in religious thinking around the world (Rozin et al., 1986; Gmelch, 1971; Malinowski, 1932; Evans-Pritchard and Gillies, 1976). This might seem puzzling from an evolutionary perspective: why should humans, a large-brained species with sophisticated cognitive adaptations (Barkow et al., 1992), systematically make mistaken inferences about cause and effect?

This quandary incorrectly assumes that superstitions cannot be viewed as practical inferential strategies in an evolutionary game against nature, rather than merely irrational quirks that interfere with our pursuit of truth (Boyer, 2018). In fact, a plausible explanation for adaptive superstition among human and non-human animals comes from the bet-hedging logic of error management theory (Haselton and Buss, 2000; McKay and Dennett, 2009). That is, simple and practical decision strategies can improve fitness in a noisy and uncertain world by managing the expected costs and benefits of two common types of error: false positives (belief in a falsehood) and false negatives (disbelief in a truth) (Trimmer et al., 2011). When presented with stimuli that might be causally linked, if the cost of a false negative is sufficiently high then natural selection will favor strategies that err on the side of committing false positives, resembling superstition (Beck and Forstmeier, 2007).

To illustrate, suppose that an organism is regularly faced with a decision task where a noisy or error-prone cue $\left(s_{1}\right.$, such as a sudden movement in the bushes) might sometimes precede a fitness-relevant outcome $\left(s_{2}\right.$, such as a lurking predator). Given an instance of $s_{1}$, the organism can either take action based on an assumption that $s_{1}$ implies $s_{2}$, or it can assume the $s_{1}$ was a false alarm (perhaps, for example, if the sudden movement was caused by wind or a harmless herbivore). If the expected benefits and/or avoided costs of taking action (e.g., fleeing from the movement) is sufficiently greater than the expected cost of taking action, regardless of error rate, then an adaptive decision policy will be prone to more false positives and fewer false negatives. Specifically, and as Foster and Kokko (2009) show, a superstitious strategy, overestimating the probability that $s_{1}$ implies $s_{2}$, should evolve when $P\left(s_{2} \mid s_{1}\right) b>c$, where $b$ is the benefit and/or avoided costs gained by taking action, and $c$ is the cost of taking action. As $P\left(s_{2} \mid s_{1}\right)$ decreases - meaning that the true conditional probability of $s_{2}$, given an event of $s_{1}$, is lower than the decision-maker's behavior would suggest - this error management strategy will appear increasingly superstitious.

One aspect of religious belief that can be understood as error management is anthropomorphism, a "reasonable illusion" to which humans seem susceptible. Guthrie (1980) has championed the view that our propensity to see minds where there are none is a deeply ancestral trait, and evolved because it pays to run (or fly or swim) whenever we detect that something unknown has agency. In other words, it's better to bet that there's a predator afoot and run all the time than to be wrong once and get eaten. Running might have its costs, but they are nothing compared to losing one's life. 
Table 4 details one possible payoff matrix for Guthrie's model (see also Bulbulia, 2004). Here, the benefits of running, $b$, have costs, $c$, while staying put when there is a predator around entails a major loss, $-b$. As long as $b>c$, running will easily outcompete the alternative.

Table 4: An example of a payoff matrix for Guthrie's Best Bet Hypothesis

\begin{tabular}{ccc}
\hline \hline & \multicolumn{2}{c}{ Predator? } \\
Run? & Yes & No \\
\hline Yes & $b-c$ & $b-c$ \\
No & $-b$ & 0 \\
\hline \hline
\end{tabular}

When this cognitive bias is expressed culturally, it can result in plausible-seeming models of the world that assume human or human-like forces are behind natural phenomena. Examples vary by culture, and they can include hidden messages, faces in the clouds (Guthrie, 1995), teleological assumptions about the natural world (Kelemen, 2004), and accusations of bad actors (including gods) behind ambiguous or unfortunate events (Gray and Wegner, 2010; Whitehouse, 2011, Jackson \& Gray, present volume).

Although these cognitive tendencies and their bet-hedging logic are clearly important contributors of religious beliefs, religious systems also include a substantial social component that goes beyond beliefs about gods, ancestors, and spirits. In fact, religious traditions, histories, and rituals can also play a key role in shaping the social dilemmas that societies face.

\section{Minds of gods and games against others}

Instead of modeling evolutionary games against nature, many early evolutionary game theorists influenced by the population genetics of Fisher (1930) also began focusing games against others. This paradigm retained its use of strategies as heritable traits (such as cooperativeness and aggression) and fitness as its metric of utility, but it emphasized population-level replicator dynamics among strategies engaging each other. An innovative analytic tool that emerged from this brand of evolutionary game theory was the evolutionary stable strategy (ESS), an applied version of the Nash equilibrium concept. An ESS refers to a strategy that, if widely adopted in the population, cannot be invaded by a mutant strategy by natural selection (Maynard Smith, 1982).

Although the inherited strategies in evolutionary game theory typically represent genetically inherited traits, they can also represent culturally transmitted responses to a recurring social dilemma. This is especially important to note about games against others, because unlike games against nature, the actors in social dilemmas are often conceptualized as members of an interactive society, rather than as a simple population of organisms.

Societies everywhere must routinely solve problems of cooperation and conflict, both among individuals and between social groups. As we saw with the Driving Game in section 1, cultural conventions can be useful for avoiding undesirable outcomes, and the stakes of a recurring social dilemma might include costly land conflicts or depleting scarce resources. Institutions often seem to supply societies with solutions to social dilemmas, and religions are no exception.

\subsection{Cooperation and supernatural punishment}

Humans and many other species are frequently altruistic, meaning that individuals cooperate with others by conferring some fitness benefit to others while forgoing a benefit to oneself. Many have argued that from an evolutionary perspective, this presents an apparent puzzle: Why should individual interests not take precedence over group interests?

The Prisoner's Dilemma (PD) formally illustrates this problem, and has been touted as "the purest expression of the conflict between individual and group interests" (McElreath and Boyd, 
2007). At its most basic, the PD includes two strategies: cooperate, $C$, and defect, $D$. Table 5 details the benefits, $b$, and $\operatorname{costs} c$ for players in a basic PD:

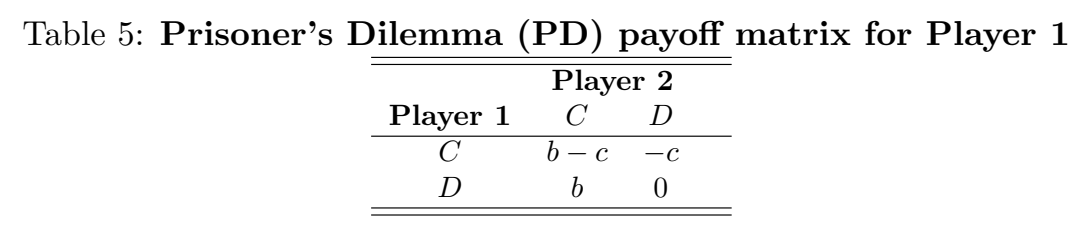

In this basic PD, it's always better to defect. To see why, imagine a population of two pure strategies: always cooperate (ALLC) and always defect (ALLD). Call the frequency of cooperators $p$. The frequency of defectors is therefore $1-p$. All individuals start with a baseline fitness of $w_{0}$. The fitness $(W)$ of ALLC is therefore defined as:

$$
\begin{aligned}
W(\mathrm{ALLC}) & =w_{0}+p(b-c)+(1-p)(-c) \\
& =w_{0}+p b-c
\end{aligned}
$$

In other words, the fitness for ALLC is its baseline fitness plus its expected fitness after interacting with another individual. With probability $p$, this individual might be a cooperator, leading to a payoff of $b-c$, or with probability $1-p$, it might be a defector, leading to a payoff of $-c$. Similarly, $W(\mathrm{ALLD})$ is defined as:

$$
\begin{aligned}
W(\mathrm{ALLD}) & =w_{0}+p b+(1-p)(0) \\
& =w_{0}+p b
\end{aligned}
$$

Since $p b>p b-c$ (so long as $c>0$ ), it follows that $W$ (ALLD) $>W$ (ALLC). In other words, defectors will always outcompete cooperators.

When a dilemma like this occurs a group setting - sometimes referred to as an $n$-person PD it can easily result a commons problem. Suppose, for example, that a community has some kind of valuable natural resource available to them, such as a common food or water supply. If this common resource is finite and overexploited, then it also carries some risk of collapse. The cooperative strategy, ensuring everyone in the community can benefit from the common resource, would be to use it with restraint. In contrast, the defect strategy would be to use the common resource without restraint, regardless of how this might withhold benefits for the rest of the community. If we suppose this hypothetical community contains ALLC and ALLD strategies (as we saw above), then defectors not only outcompete cooperators: They also virtually guarantee that the common resource will collapse, a consequence of everyone overusing it out of narrow self-interest. This result is called a tragedy of the commons (Hardin, 1968).

This clearly is not the world in which we live. Human societies routinely cooperate with each other to sustain common resources, even where they are vulnerable to a temptation to defect. How, then, do societies reliably shift their incentives in a way that "solves" the PD by favoring widespread cooperation, rather than widespread defection? There are a few ways to manage this dilemma, each of which make the prospect of defection less inviting.

One simple solution would be to impose a threat of substantial costs, or punishment, on defectors (Ostrom, 1990). Let's call the cost of being punished for defection $c_{d}$. As long as the expected cost of defection, $\mathbf{E}\left(c_{d}\right)$, outweighs the cost of cooperation ( $c$, from table 5), then $p b-c>p b-\mathbf{E}\left(c_{d}\right)$. This means that, after including punishment for defection, cooperators outperform defectors (because $W(\mathrm{ALLC})>W(\mathrm{ALLD}))$. The reason we use the expected cost of punishment is to explicitly state a critical ingredient of deterring possible defection through punishment: The looming threat of punishment, in the event of defection, must be sufficiently credible. We can write the expected cost of defection as $\mathbf{E}\left(c_{d}\right)=c_{d} p\left(c_{d}\right)$, conveniently specifying the cost of being punished for defection, $c_{d}$, and the probability of being punished for defection, $p\left(c_{d}\right)$. 
Credible threats of punishment for defection not only require enforcing cooperative rules, but an ability to monitor behavior for violations of those rules. We can illustrate this by considering how a PD scenario, such as food sharing, might play out in a very small community. In such a context, behaviors and reputations are fairly easy to monitor, so punishments, such as retaliation or avoidance, would be easy to mete out when selfish behaviors occur. In other words, $p\left(c_{d}\right)$ is high. If $c_{d}$ is also sufficiently high, then cooperation becomes a favorable strategy.

Now consider a contrasting example: large-scale societies and market economies, where individuals make frequent one-shot, impersonal exchanges with unrelated strangers. Here, $p\left(c_{d}\right)$ is lower than it was in our previous example because credible threats of punishment for defection are more difficult to achieve on a larger scale. This means that, all else equal, $\mathbf{E}\left(c_{d}\right)$ is relatively low, and our PD is much more likely to favor widespread defection. People can seemingly "cheat" with impunity when selling a used car, tipping for a service, or returning a lost wallet - especially when they need not worry about reputational consequences and an absence of future interactions. Commons problems, such as donating to a public radio station, shouldn't fare much better. If anonymous freeriders could maximize their expected payoffs by receiving the results of the generous work of many others, all without making a contribution, then how did large-scale societies ever emerge?

Moralizing gods - gods who are omniscient, care about moral violations, and threaten to punish defectors - might help answer this question (Lang et al., 2019; Norenzayan et al., 2016, see Purzycki and McKay, present volume). In a PD, widespread belief in moralizing gods can shift incentives in a way that favors cooperation, resulting from a fear of supernatural punishment (Johnson and Bering, 2006; Schloss and Murray, 2011) - even where defection might be otherwise beneficial, like we'd see in a one-shot and anonymous interaction (Johnson, 2015). This is a potentially powerful solution to the PD; if we conceptualize belief in supernatural punishment as a culturally inherited strategy, then it will spread alongside other forms of punishment for defection in formal evolutionary models (Lane, 2018), such as the retaliatory strategies that are possible in smaller-scale settings. Hence, assuming communication is possible, we can construe appeals to supernatural punishment as attempts to control other people's social behaviors, ultimately motivating them to behave in ways that benefit others (Cronk, 1994; Fitouchi and Singh, 2022). The looming threat of supernatural punishment has the potential to expand people's cooperative circles, introducing mutually beneficial solutions to cooperative problems that would have been difficult for individuals to consider otherwise. We revisit this point below.

\subsection{Coordination and signaling trust}

Despite the narrowly selfish behaviors that a standard PD motivates, human life is filled with scenarios where it is mutually beneficial for individuals to cooperate, especially in the long-term. The Stag Hunt captures such scenarios. It imagines two hunters who must decide to either help each other pursue a large stag, the success of which requires the effort of two people cooperating, or to each individually hunt small hares, which does not require any cooperative effort. Hence, a standard Stag Hunt game has two strategies: players can hunt hares, or they can hunt stags. The mutually cooperative outcome (hunting stags) is in the interests of both individuals, so long as they can commit to working toward the same outcome together (Skyrms, 2003). Table 6 details the benefits in a standard Stag Hunt gained from hunting stags, $S$, vs. the benefits of hunting hares, $h$, where $h<S$.

Table 6: Payoff matrix for the Stag Hunt game.

\begin{tabular}{ccc}
\hline \hline & \multicolumn{2}{c}{ Player 2 } \\
Player 1 & Stag & Hares \\
\hline Stag & $S, S$ & $0, h$ \\
Hares & $h, 0$ & $h, h$ \\
\hline \hline
\end{tabular}


As the payoff matrix suggests, the Stag Hunt introduces a coordination problem that is akin to the Driving Game in section 1: Each player's best response is to do what the other player is doing. Hunting hares yields a low but certain payoff, regardless of what the other actor does. Hunting stags yields a high payoff to both players, but only if the players coordinate doing so together otherwise, the stag hunter who took a risk on a partner who chose the hare gets nothing. This dilemma between high-risk/high-reward stags vs. low-risk/low-reward hares implies that rational utility-maximizers would be wise to choose the risk-averse hare strategy if unsure what other players will do. If they can trust that others will commit to stag hunting, however, then they should also commit to stag hunting. Cooperators can mutually benefit if they find partners whose behaviors are predictably cooperative, meaning that in the Stag Hunt, communicating trustworthiness is critical.

In many ways, the Stag Hunt game best captures the nature of a social contract. Players need to somehow collectively agree to move from a suboptimal convention (everyone hunts hares) to a more optimal one (everyone hunts stags). In the absence of communication and trust, getting everyone to make this commitment is difficult. Religious beliefs and institutions can improve the prospects for doing so, however, coordinating sustainable and unconditional solutions to a cooperative dilemma (Bulbulia, 2012; Shaver and Bulbulia, 2016).

To see an example of this, let's return to supernatural punishment beliefs. Shared beliefs in a moralizing god inspire prosociality among distant strangers, often from distant regions and otherwise different cultural backgrounds (Lang et al., 2019). Nevertheless, cooperative believers are clearly vulnerable to exploitation by non-believers, because non-believers can instill trust by feigning belief before defecting against a believer. How, then, can believers reliably identify who among them can be trusted to also believe in supernatural punishment (and thus, to share the same incentives that motivate cooperation)? If there were a way for strangers to honestly signal their shared identities, and to communicate their cooperative intentions to each other, then this would greatly improve our prospects of expanding cooperation beyond kin and other tight-knit networks.

Religious rituals are useful for improving within-group coordination, precisely because they can signal common commitments to a religious identity (Sosis and Alcorta, 2003). Communities with shared religious identities often have ritual obligations that entail opportunity costs that serve as hard-to-fake signs of commitment to the ingroup (Irons, 2001), because for true believers, the perceived costs of these obligations are lower than they are for non-believers (Sosis, 2003). This view conceptualizes ritual as a form of communication that improves the prospects of trust and cooperation among coreligionists. Commitment displays might have assisted the spread of Islam in Africa among long-distance trade routes, for example, where conflicts of interest among resource holders and traveling merchants can easily lead to defection by the traveling merchants (a principalagent problem; see North, 1990). Both parties can instill trust, however, by credibly communicating (e.g., through daily prayer, Ramadan fasting, and the annual Hajj) that they are each committed to honoring a mutually beneficial code of conduct with supernatural sanctions - despite their geographic and cultural differences (Ensminger, 1997).

\subsection{Engineering an ecosystem}

Religions also help coordinate solutions to the "us vs. nature" problems that societies face, beneficially shaping how people engage their environments. A classic example of this is the water temple system used for coordinating watering schedules among Balinese rice farmers (Lansing and Kremer, 1993). Rice farming collectives called subaks must manage two competing ecological pressures: crop pests and water availability. Crop pests affect everyone equally, whereas water availability is limited for downstream subaks, depending on how much water is used first by upstream subaks.

A simplified version of this scenario is modeled as a game between an upstream subak and a downstream subak in table 7 , where payoffs represent crop yields for each subak. The upstream subak and the downstream subak can choose to water their fields at one of two times of year (let's call them Time A and Time B). 
If subaks coordinate by watering their fields at the same time, then they mutually benefit by reducing crop pests, but the upstream subak will deplete the water available to the downstream subak, reducing the downstream subak's crop yield by $d$. The downstream subak can avoid losses due to water depletion by not coordinating their timing with the upstream subak, but then crop pests will reduce the crop yields for both subaks by $r$.

Table 7: Payoff matrix for the coordination problem faced by upstream and downstream subaks in Bali.

\begin{tabular}{ccc}
\hline \hline & \multicolumn{2}{c}{ Downstream } \\
Upstream & Time A & Time B \\
\hline Time A & $1-d, 1$ & $1-r, 1-r$ \\
Time B & $1-r, 1-r$ & $1,1-d$ \\
\hline \hline
\end{tabular}

The resulting coordination game incentivizes the upstream subak to always use water at the same time as the downstream subak. The downstream subak should only want to coordinate its timing with the upstream subak if crop pest damages outweigh the costs of water depletion $(r>d)$. If the upstream subak depletes the water so much that the downstream subak would prefer crop pest damages to water depletion $(r<d)$, then the downstream subak should be motivated to not coordinate. Hence, the dilemma for the upstream subak is to not only ensure that they coordinate their timing of water use with the downstream subak, but also to minimize their water use in a way that keeps the downstream subak motivated to coordinate (Lansing and Miller, 2005).

As we've seen throughout this chapter, communicating and forming conventions can greatly improve the prospects for resolving this type of dilemma. In Bali, these improvements are supplied by the water temple system, where subaks make coordination pacts with mutual displays of deference to deities. In reality, and scaled beyond the two-player simplification we've discussed here, this subak water temple system is a complex network that has sustained coordination among subaks for centuries - not through punishment, but through a signaling system that improves communication among parties with overlapping interests (Lansing and Fox, 2011).

Another example of a religious system improving a local coordination problem can be found with the Australian Martu, whose landscape burning practices increase the local plant biodiversity. This, in turn, leads to high and sustained numbers of small herbivores that attract the monitor lizards who prey on them. These lizards not only provide food resources for the Martu, but hunting them has downstream social effects like the establishment and strengthening of food sharing relationships (Bliege Bird and Power, 2015). Controlled burning practices are an important group effort in this region, and the regions where burning is practiced are the regions where lizard hunting is relatively successful (Bliege Bird et al., 2013).

We can frame Martu burning practices as a Stag Hunt, where the payoffs of collective burning can ensure the individual- and group-beneficial payoff of high lizard numbers, $L$. If few people participate then the burning practices might be ineffective despite the effort expended by the participant, $c$, and if no one participates then each individual must hunt from a small lizard population, $l$, where $L>l$. See table 8 .

Table 8: Payoff matrix for Player 1 in a Martu burning dilemma.

\begin{tabular}{ccc}
\hline \hline & \multicolumn{2}{c}{ Player 2 } \\
Player 1 & Burn & Don't burn \\
\hline Burn & $L$ & $l-c$ \\
Don't burn & $l$ & $l$ \\
\hline \hline
\end{tabular}

This coordination problem is improved by the religious beliefs associated with the burning practices, because Martu associate them with Jukurrpa, an idea that a failure to participate in 
burning practices would result in the end of the world. By incentivizing burning over not burning, this belief can serve as a mechanism for helping individuals converge toward, and remain stably committed to, the coordination solution of this locally important social dilemma (Bird et al., 2016).

\subsection{Conflict over territory and resources}

While coordination is clearly important for resource maintenance, a scarcity of resources often leads to conflict, especially when linked to a desirable territory. The Hawk-Dove game models this type of scenario, where two strategies compete for a resource with some fitness benefit, $v$. The Hawk strategy, $H$, always fights for the resource and never retreats, whereas the Dove, $D$, strategy might display a threat, but will retreat if attacked.

If a Hawk encounters a Dove, then the Dove will retreat uninjured, and the Hawk will get the resource without challenge. If two Doves meet in a given encounter, then the resource will either be randomly taken by one of the individuals, or if possible, evenly shared. If two Hawks meet, however, they will each incur a fighting cost $c$. Assuming equal fighting abilities among two randomly selected Hawks in a population, a Hawk can expect to win (and take the resource) in half of its encounters.

These payoffs are shown in terms of fitness benefits, $v$, and costs, $c$, to Hawk and Dove strategies in table 9:

Table 9: Hawk-Dove payoff matrix for Player 1.

\begin{tabular}{ccc}
\hline \hline & \multicolumn{2}{c}{ Player 2 } \\
Player 1 & $H$ & $D$ \\
\hline$H$ & $\frac{v-c}{2}$ & $v$ \\
$D$ & 0 & $\frac{v}{2}$ \\
\hline \hline
\end{tabular}

Are either of these strategies stable enough to withstand invasion from the other? To answer this, let us create our own population. Assume that in this case, Doves are almost entirely absent ( $p$, the frequency of encountering a Hawk, is approximately 1). Hawks primarily interact with other Hawks. Their approximate expected fitness, $W(H)$, then, would be

$$
\begin{aligned}
W(H) & \approx w_{0}+(1) V(H \mid H)+(0) V(H \mid D) \\
& \approx w_{0}+\frac{v-c}{2}
\end{aligned}
$$

In other words, the expected fitness of a Hawk would be their baseline fitness, $w_{0}$, the payoffs of interacting with another Hawk, $\frac{v-c}{2}$ times the probability of interacting with another Hawk (remember, in our created world, it is $\approx 1$ ), and the payoff of interacting with a Dove times the likelihood of interacting with a Dove (in this case, 0 ). If we introduce a Dove into this world, its expected payoff is thus:

$$
\begin{aligned}
W(D) & \approx w_{0}+V(D \mid H) \\
& \approx w_{0}+0
\end{aligned}
$$

What, then, is required to ensure that $W(H)<W(D)$ ? We can answer this question by plugging in what we know to the above equations, which simplifies to $v<c$. What this means is that Doves can only invade the population when fighting costs outweigh the value of the resource.

Monitoring and enforcing the borders of a given territory can be costly and difficult, and it might nevertheless lead to costly conflicts over contested land or resources. One way to curb these types of risks and tensions among communities would be to encourage widespread beliefs in locally powerful deities who monitor and enforce respect for existing territories.

Consider, as an example, the Tyvan spirit-masters who are associated with ritual structures that people approach with reverence and prayer offerings. Each spirit-master cares about its local territory and its natural resources, and is believed to be knowledgeable about the goings on in its 
territory. Spirit-masters are angered over-exploiting resources, violations of which are believed to be met with devastating consequences (Purzycki, 2011, 2013). These beliefs and their attendant practices can shift incentives in a way that favors preserving resources that are otherwise at risk of overexploitation (Purzycki, 2016). This clearly resembles a solution to an $n$-person PD, discussed above, where a common resource is susceptible to widespread defection and the incentives are tilted toward widespread cooperation.

Another aspect of these gods' concerns worth emphasizing is that the ritual requirements linked to territory structures might serve an additional function. By indicating spirits present at a given border, and by requiring passersby to acknowledge their presence, individuals are expected to respectfully engage the existing border structures in the region. The scenario resembles a HawkDove game, because whereas different camps can share the land with a mutual "dovish" respect for existing territories, they might also be tempted to engage in a risky and "hawkish" expansion into new territories, particularly if more livestock requires more grazing land. In this context, ritual structures and their attendant spirit masters can motivate a mutual dovish respect through threats of supernatural punishment, specifically by increasing the perceived fighting costs in a way that favors a dovish strategy, where $v<c$. Similar to the moralizing gods example we saw above, this shift in incentives is accompanied by a shift in the social signals that people can deploy to instill trust among their coreligionists. Indeed, people who conform to the ritual expectations of the local spirit masters tend to inspire trust among observers (Purzycki and Arakchaa, 2013).

\section{Future directions and open questions}

Anthropologists have long recognized that traditional institutions tend to be useful for dealing with locally important dilemmas (Rappaport, 1968; Steward, 1972), but explaining this pattern has been more challenging. How do traditional institutions, such as religions, give rise to adaptive practices among diverse social and ecological settings? Explanations invoking processes like transmitted culture and cognitively attractive ideas are undoubtedly important for understanding religion (Jackson \& Gray, present volume), because religious beliefs are intuitive - for good evolutionary reasons, as we discussed in section 2 - and they are also socially learned.

And yet, if we want a complete explanation of how religious systems can adjust to a complex and dynamic environment, then we must also carefully consider: (1) how the individuals will attend to their expected payoffs in a given environment, and (2) when and how religious beliefs about gods' minds, and corollary practices such as rituals, impact the scope for improving coordination and reducing costly conflicts (Bendixen and Purzycki, 2020). As we've outlined in this chapter, game theory provides us with a powerful formal framework for doing so. Through its lens, we can clarify how and why religious traditions are often linked to behaviors that might not be incentivized on an individual level.

The examples we've discussed through a game theoretic lens are nevertheless, in many cases, post hoc interpretations of social and environmental dilemmas. Game theoretic models are simple and useful tools for predicting behavior based on a minimal set of assumptions, but their role in explaining how religious traditions can resolve social dilemmas will be substantially improved as their empirical predictions are put to the test in future research. To do this, rich ethnographic descriptions of societies' challenges "on the ground" should inform which payoffs individuals are likely to deem relevant, what resulting conflicts of interest could threaten their social or ecological stability, and how we would predict religious systems to improve the prospects of reducing such conflicts. 


\section{Acknowledgments}

Both authors thank the Aarhus University Research Foundation for support. Purzycki expresses thanks to the students in his Minds of Gods classes for their discussions and input, and both authors thank Theiss Bendixen and an anonymous reviewer for helpful comments that greatly improved this chapter. Thanks to Steven Brams and Joseph Bulbulia for their suggestions.

\section{References}

Barkow, J. H., Cosmides, L., and Tooby, J. (1992). The Adapted Mind: Evolutionary Psychology and the Generation of Culture. Oxford University Press, USA.

Beck, J. and Forstmeier, W. (2007). Superstition and belief as inevitable by-products of an adaptive learning strategy. Human Nature, 18(1):35-46.

Bendixen, T. and Purzycki, B. G. (2020). Peering into the minds of gods: What cross-cultural variation in gods' concerns can tell us about the evolution of religion. Journal for the Cognitive Science of Religion, 5(2):142-165.

Binmore, K. (2011). Natural Justice. Oxford Univ. Press, Oxford.

Binmore, K. G. (1994). Game Theory and the Social Contract. V. 2: MIT Press Series on Economic Learning and Social Evolution. MIT Press, Cambridge, Mass.

Bird, D. W., Bird, R. B., Codding, B. F., and Taylor, N. (2016). A landscape architecture of fire: Cultural emergence and ecological pyrodiversity in australia's western desert. Current Anthropology, 57(S13):S65-S79.

Bliege Bird, R. and Power, E. (2015). Prosocial signaling and cooperation among martu hunters. Evolution and Human Behavior, 36(5):389-397.

Bliege Bird, R., Tayor, N., Codding, B. F., and Bird, D. W. (2013). Niche construction and Dreaming logic: Aboriginal patch mosaic burning and varanid lizards (Varanus gouldii ) in Australia. Proceedings of the Royal Society B: Biological Sciences, 280(1772):20132297.

Bossaerts, P. and Murawski, C. (2017). Computational complexity and human decision-making. Trends in Cognitive Sciences, 21(12):917-929.

Boyer, P. (2018). Minds Make Societies: How Cognition Explains the World Humans Create. Yale University Press, New Haven ; London, 1st edition edition edition.

Brams, S. J. (1982). Belief in god: A game-theoretic paradox. International Journal for Philosophy of Religion, 13(3):121-129.

Brams, S. J. (1983). Superior beings: If they exist, how would we know? Springer, New York.

Brams, S. J. (2018). Divine games: Game theory and the undecidability of a superior being. MIT Press.

Bulbulia, J. (2004). The cognitive and evolutionary psychology of religion. Biology and philosophy, 19(5):655-686.

Bulbulia, J. (2012). Spreading order: religion, cooperative niche construction, and risky coordination problems. Biology 83 Philosophy, 27(1):1-27. 
Cronk, L. (1994). Evolutionary theories of morality and the manipulative use of signals. Zygon, 29(1):81-101.

Doyle, A. C. (2003). Sherlock Holmes: The Complete Novels and Stories: Volumes I and II. Bantam Classics, New York.

Ensminger, J. (1997). Transaction Costs and Islam: Explaining Conversion in Africa. Journal of Institutional and Theoretical Economics (JITE) / Zeitschrift für die gesamte Staatswissenschaft, 153(1):4-29.

Evans-Pritchard, E. E. and Gillies, E. (1976). Witchcraft, Oracles, and Magic among the Azande. Clarendon Press, Oxford, abridged with an introd. by eva gillies edition.

Fisher, R. A. (1930). The genetical theory of natural selection. Oxford University Press.

Fitouchi, L. and Singh, M. (2022). Supernatural punishment beliefs as cognitively compelling tools of social control. Current opinion in psychology, 44:252-257.

Foster, K. R. and Kokko, H. (2009). The evolution of superstitious and superstition-like behaviour. Proceedings of the Royal Society B: Biological Sciences, 276(1654):31-37.

Gigerenzer, G., Hertwig, R., and Pachur, T. (2011). Heuristics. Oxford University Press.

Gmelch, G. (1971). Baseball magic. Trans-action, 8(8):39-41.

Gray, K. and Wegner, D. M. (2010). Blaming god for our pain: Human suffering and the divine mind. Personality and Social Psychology Review, 14(1):7-16.

Guthrie, S. (1980). A cognitive theory of religion. Current Anthropology, 21(2):181-203.

Guthrie, S. E. (1995). Faces in the Clouds: A New Theory of Religion. Oxford University Press USA, New York, 1st edition edition.

Hacking, I. (1972). The logic of pascal's wager. American Philosophical Quarterly, 9(2):186-192.

Hardin, G. (1968). The Tragedy of the Commons. Science, 162(3859):1243-1248.

Haselton, M. G., Bryant, G. A., Wilke, A., Frederick, D. A., Galperin, A., Frankenhuis, W. E., and Moore, T. (2009). Adaptive rationality: An evolutionary perspective on cognitive bias. Social Cognition, 27(5):733-763.

Haselton, M. G. and Buss, D. M. (2000). Error management theory: a new perspective on biases in cross-sex mind reading. Journal of personality and social psychology, 78(1):81.

Irons, W. (2001). Religion as a hard-to-fake sign of commitment. Evolution and the capacity for commitment, 292309.

Johnson, D. (2015). God Is Watching You: How the Fear of God Makes Us Human. Oxford University Press, New York, 1 edition edition.

Johnson, D. and Bering, J. (2006). Hand of God, mind of man: Punishment and cognition in the evolution of cooperation. Evolutionary Psychology, 4:219-233.

Kelemen, D. (2004). Are children "intuitive theists"? Reasoning about purpose and design in nature. Psychological science, 15(5):295-301.

Kokko, H. (2007). Modelling for field biologists and other interesting people. Cambridge University Press. 
Lane, J. (2018). Strengthening the supernatural punishment hypothesis through computer modeling. Religion, Brain \& Behavior, 8(3):290-300.

Lang, M., Purzycki, B. G., Apicella, C. L., Atkinson, Q. D., Bolyanatz, A., Cohen, E., Handley, C., Kundtová Klocová, E., Lesorogol, C., Mathew, S., McNamara, R. A., Moya, C., Placek, C. D., Soler, M., Vardy, T., Weigel, J. L., Willard, A. K., Xygalatas, D., Norenzayan, A., and Henrich, J. (2019). Moralizing gods, impartiality and religious parochialism across 15 societies. Proceedings of the Royal Society B: Biological Sciences, 286(1898):20190202.

Lansing, J. S. and Fox, K. M. (2011). Niche construction on bali: the gods of the countryside. Philosophical Transactions of the Royal Society B: Biological Sciences, 366(1566):927-934.

Lansing, J. S. and Kremer, J. N. (1993). Emergent properties of Balinese water temple networks: Coadaptation on a rugged fitness landscape. American Anthropologist, 95(1):97-114.

Lansing, J. S. and Miller, J. (2005). Cooperation, games, and ecological feedback: some insights from bali. Current Anthropology, 46(2):328-334.

Lewis, D. (1969). Convention: A philosophical study. John Wiley \& Sons.

Lewontin, R. C. (1961). Evolution and the theory of games. Journal of theoretical biology, 1(3):382403.

Malinowski, B. (1932). Argonauts Of The Western Pacific. George Routledge And Sons, Limited.

Maynard Smith, J. (1982). Evolution and the Theory of Games. Cambridge University Press, Cambridge ; New York.

McElreath, R. and Boyd, R. (2007). Mathematical Models of Social Evolution: A Guide for the Perplexed. University of Chicago Press, Chicago ; London.

McKay, R. T. and Dennett, D. C. (2009). The evolution of misbelief. Behavioral and Brain Sciences, $32(6): 493-510$

McNamara, J. M. and Leimar, O. (2020). Game theory in biology: concepts and frontiers. Oxford University Press, USA.

Nash, J. F. (1950). Equilibrium points in n-person games. Proceedings of the national academy of sciences, 36(1):48-49.

Norenzayan, A., Shariff, A. F., Gervais, W. M., Willard, A. K., McNamara, R. A., Slingerland, E., and Henrich, J. (2016). The cultural evolution of prosocial religions. Behavioral and Brain Sciences, 39:e1.

North, D. C. (1990). Institutions, Institutional Change, and Economic Performance. The Political Economy of Institutions and Decisions. Cambridge University Press, Cambridge ; New York.

Ostrom, E. (1990). Governing the Commons: The Evolution of Institutions for Collective Action. Cambridge University Press, Cambridge ; New York, 1st edition edition.

Prakash, C., Stephens, K. D., Hoffman, D. D., Singh, M., and Fields, C. (2021). Fitness beats truth in the evolution of perception. Acta Biotheoretica, 69(3):319-341.

Purzycki, B. G. (2011). Tyvan cher eezi and the socioecological constraints of supernatural agents' minds. Religion, Brain \& Behavior, 1(1):31-45. 
Purzycki, B. G. (2013). Toward a cognitive ecology of religious concepts: An example from the tyva republic. Journal for the Cognitive Science of Religion, 1(1):99-120.

Purzycki, B. G. (2016). The Evolution of Gods' Minds in the Tyva Republic. Current Anthropology, 57(S13):S88-S104.

Purzycki, B. G. and Arakchaa, T. (2013). Ritual behavior and trust in the tyva republic. Current Anthropology, 54(3):381-388.

Purzycki, B. G. and Sosis, R. (2022). Religion Evolving; The Dynamics of Culture, Cognition, and Ecology. Equinox, Sheffield.

Rappaport, R. A. (1968). Pigs for the Ancestors: Ritual in the Ecology of a New Guinea People. Waveland Press, Long Grove, Ill, 1. ed edition.

Rozin, P., Millman, L., and Nemeroff, C. (1986). Operation of the laws of sympathetic magic in disgust and other domains. Journal of Personality and Social Psychology, 50(4):703-712.

Schloss, J. P. and Murray, M. J. (2011). Evolutionary accounts of belief in supernatural punishment: A critical review. Religion, Brain \& Behavior, 1(1):46-99.

Shaver, J. H. and Bulbulia, J. A. (2016). Signaling theory and religion. Religion: Mental Religion, pages $101-117$.

Skyrms, B. (2003). The Stag Hunt and the Evolution of Social Structure. Cambridge University Press, Cambridge.

Sosis, R. (2003). Why aren't we all Hutterites? Human nature, 14(2):91-127.

Sosis, R. and Alcorta, C. (2003). Signaling, solidarity, and the sacred: The evolution of religious behavior. Evolutionary Anthropology: Issues, News, and Reviews, 12(6):264-274.

Steward, J. H. (1972). Theory of Culture Change: The Methodology of Multilinear Evolution. University of Illinois Press.

Trimmer, P. C., Houston, A. I., Marshall, J. A. R., Mendl, M. T., Paul, E. S., and McNamara, J. M. (2011). Decision-making under uncertainty: Biases and Bayesians. Animal Cognition, $14(4): 465-476$.

Von Neumann, J. and Morgenstern, O. (1953). Theory of Games and Economic Behavior. Princeton Classic Editions. Princeton University Press, Princeton, N.J. ; Woodstock, 60th anniversary ed edition.

Whitehouse, H. (2011). The coexistence problem in psychology, anthropology, and evolutionary theory. Human Development, 54(3):191-199.

Yamagishi, T. and Suzuki, N. (2009). An institutional approach to culture. In Evolution, Culture and the Human Mind. Psychology Press. 